\title{
Shape versus Size: Improved Understanding of the Morphology of Brain Structures
}

\author{
${ }^{1,2}$ Guido Gerig, ${ }^{1}$ Martin Styner, ${ }^{3}$ Martha E. Shenton, ${ }^{2}$ Jeffrey A. Lieberman \\ ${ }^{1}$ Department of Computer Science, UNC, Chapel Hill, NC 27599, USA \\ ${ }^{2}$ Department of Psychiatry, UNC, Chapel Hill, NC 27599, USA \\ ${ }^{3}$ Department of Psychiatry, VAMC-Brockton, Harvard Medical School, Boston, USA \\ gerig@cs.unc.edu, martin styner@ieee.org
}

\begin{abstract}
Standard practice in quantitative structural neuroimaging is a segmentation into brain tissue, subcortical structures, fluid space and lesions followed by volume calculations of gross structures. On the other hand, it is evident that object characterization by size does only capture one of multiple aspects of a full structural characterization. Desirable parameters are local and global parameters like length, elongation, bending, width, complexity, bumpiness and many more. In neuroimaging research there is increasing evidence that shape analysis of brain structures provides new information which is not available by conventional volumetric measurements. This motivates development of novel morphometric analysis techniques answering clinical research questions which have been asked for a long time but which remained unanswered due to the lack of appropriate measurement tools. Challenges are the choice of biologically meaningful shape representations, robustness to noise and small perturbations, and the ability to capture the shape properties of populations that represent natural biological shape variation. This paper describes experiments with two different shape representation schemes, a finescale, global surface characterization using spherical harmonics, and a coarsely sampled medial representation (3D skeleton). Driving applications are the detection of group differences of amhygdala-hippocampal shapes in schizophrenia and the analysis of ventricular shape similarity in a mono/dizygotic twin study. The results clearly demonstrate that shape captures information on structural similarity or difference which is not accessible by volume analysis. Improved global and local structure characterization as proposed herein might help to explain pathological changes in neurodevelopment/neurodegeneration in terms of their biological meaning.
\end{abstract}

\section{Introduction}

In-vivo imaging studies of brain structures have provided valuable information about the nature of neuropsychiatric disorders including neurodegenerative diseases and/or disorders of abnormal neurodevelopment. Deformities in brain structure in Alzheimer's and Huntington's disease are believed to be due the effects of the disease process in adulthood after a period of normal neurodevelopment, while diseases like Autism and Fragile $\mathrm{X}$ syndrome are thought to involve abnormal neurodevelopment which give rise to the symptoms of the illness. Schizophrenia, on the other hand, is 
often subject to conflicting hypotheses about the cause and temporal evolution of the neuropathologic features of the disorder. Structural imaging studies so far have most often focused on volumetric assessment of brain structures, for example full brain or hemispheric gray and white matter, ventricular volume and hippocampus. Increasing evidence for structural changes in small subregions and parts of structures drive development of new structure analysis techniques. Wang [Wang 2000] found that while hippocampal volume did not discriminate schizophrenia groups from control groups, shape measurements did provide a distinct group separation. The paper further discusses that summary comparisons of whole structures ignores the possibility of detecting regional differences. Csernansky [Csernansky et al., 1998] suggests that a full characterization of neuroanatomical abnormalities will increase our understanding of etiology, pathogenesis, and pathophysiology of schizophrenia. Results show that the analysis of hippocampal shape discriminates schizophrenia and control subjects with greater power than volumetry [see also Haller et al., 1996,1997]. [Suddath et al., 1990] found smaller anterior hippocampi in affected vs. unaffected MZ twins. All these studies advocate new morphometry techniques to study shape rather than gross volume and to provide quantitative measures that are not only statistical significant but also neuroanatomically relevant and intuitive.

\section{Shape Modeling}

Surface-based shape representation: We applied a technique for surface parametrization that uses expansion into a series of spherical harmonics (SPHARM) [Brechbuehler, 1995, Szekely et al., 1996, Kelemen, 1999]. The development parallels the seminal work of Cootes \& Taylor [Cootes et al., 1995] on active shape models but is based on a parametric object description (inspired by Staib and Duncan [Staib, 1996]) rather than a point distribution model. SPHARM is a global parametrization method, i.e. small local changes can affect all parameters. It allows simple alignment of structures and gives a good initial point-to-point correspondence.

Medially-based surface shape representation: As an alternative to surface-based global shape representation, the UNC research group is working on a 3D skeletal representation with coarse to fine sampling (Pizer, 1999, Yushkevich, 2001, Styner, 2000, 2001). The medial shape representation provides locality of width and bending on a hierarchy of scales. Parameters derived from the medial representation are more intuitive than Fourier coefficients and will help to develop shape descriptions expressed in natural language terms.

\section{Applications in Neuroimaging}

The following subsections describe applications of surface-based and medially-based shape representation methods in two clinical studies. 


\subsection{Statistical Analysis of Amygdala-Hippocampal Asymmetry in Schizophrenia}

We studied the asymmetry of the hippocampal complex for a group of 15 controls and 15 schizophrenics (collaboration with M. E. Shenton, Harvard). Asymmetry was assessed by segmentation using deformable models (Kelemen, 1999), by flipping one object across the midsagittal plane, by aligning the reference and the mirrored object using the coordinates of the first ellipsoid, and by calculating the MSD between the two surfaces (Fig.1).

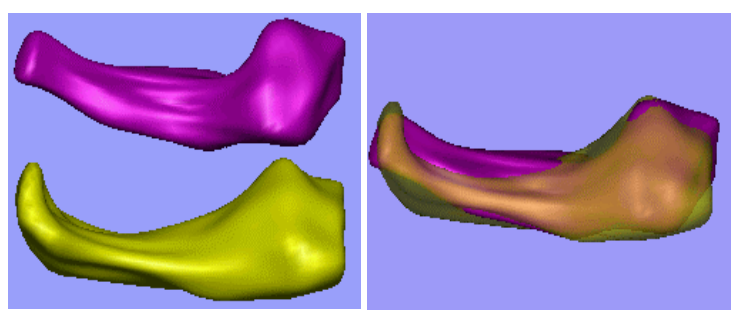

Fig. 1. Analysis of amygdalahippocampal left/right asymmetry. The left hippocampal complex is mirrored, aligned and overlaid (right) to calculate the mean square distance between surfaces.

As shape difference measures can be largely influenced by object size differences, we normalized all the objects by volume. The statistical analysis can be based on the single features $\mathrm{L} / \mathrm{R}$ volume asymmetry or $\mathrm{L} / \mathrm{R}$ shape difference, but also by combining both values (Table 1, Fig.2). Similarly to published results [Csernansky et al., 1998], we found that the volume asymmetry expressed by $(|\mathrm{L}-\mathrm{R}|) /(\mathrm{L}+\mathrm{R})$ did not discriminate the groups well if we consider classification $(70 \%)$, although the group difference was significant $(\mathrm{P}<0.0032)$. Using both shape and volume in a composite analysis (Fig. 2 right) results in a correct classification of 13 out of 15 for both groups and thus in an overall classification rate of $87 \%$. We used a support vector machine (SVM) method (Vapnik, 1998). Unbiased classification performance was obtained by leave-one-out tests.

\begin{tabular}{|l|l|l|l|}
\hline & Volume & Shape & Volume \& Shape \\
\hline & rel. L-R diff. & MSD surfaces & \\
\hline Student t-test & $\mathrm{F}=10.4, \mathrm{P}<0.0032$ & $\mathrm{~F}=5.0, \mathrm{P}<0.0335$ & $\mathrm{~F}=11.19, \mathrm{P}<0.0024$ \\
\hline Classification & $70 \%$ & $73 \%$ & $87 \%(\mathrm{SVM})$ \\
\hline
\end{tabular}

Table 1. Statistics of left/right asymmetry analysis of the amygdala-hippocampal complex for volume and shape characterization. Classification is poor and not much better than a guess for volume and shape only, whereas the combined analysis shows a significantly improved classification rate despite of the small numbers of 15 controls and 15 schizophrenics.

\subsection{Similarity of Lateral Ventricles in Mono-/Dizygotic Twin Study}

The study of identical (monzygotic, MZ) and nonidentical twins (dizygotic, DZ) provides an excellent opportunity to study similarities and differences of the morphology with respect to a genetic effect. Moreover, discordant twins, i.e. one subject affected by illness and the related subject healthy, allow further insight into the genetic factor of illness [Suddath, 1990]. We have access to an MRI twin study of 

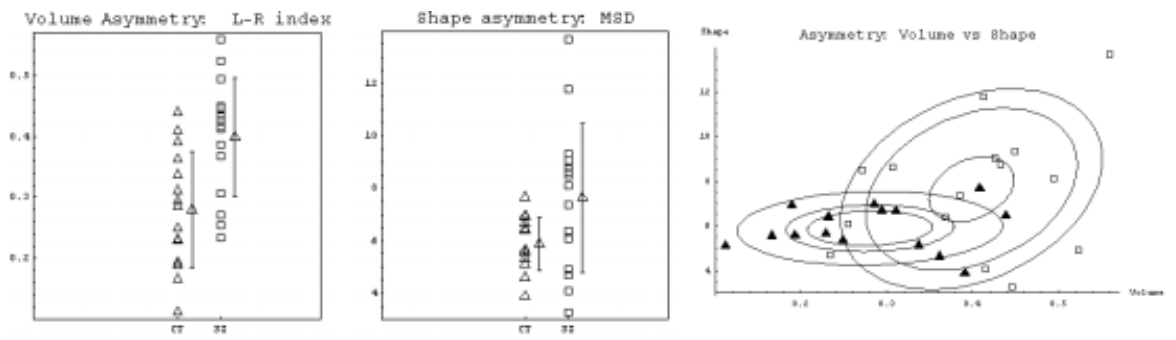

Fig. 2. Statistics of volume, shape and volume\&shape lateralization of the amygdalahippocampal complex in schizophrenia. The left figure shows the statistics of the volume asymmetry index $(\mid \mathrm{R}-\mathrm{L} / /(\mathrm{R}+\mathrm{L}))$ for the control and schizophrenics groups. The middle figure illustrates the shape difference (mean square difference between corresponding surface points, MSD) for both groups after volume normalization of each structure. The figure to the right shows a combined analysis of volume and shape asymmetry, illustrated as a two-dimensional feature space. Controls and schizophrenics are marked by dark triangles and open squares, respectively, with overlay of quartile ellipsoids.

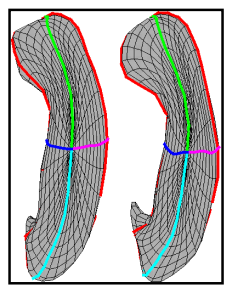

T1A-L / T1B-L

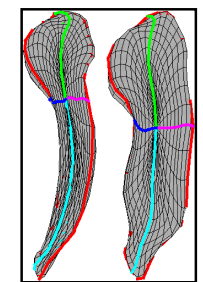

T10A-L / T10B-L T10B-R / T10A-R

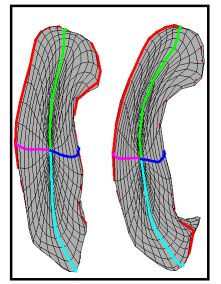

T1B-R / T1A-R

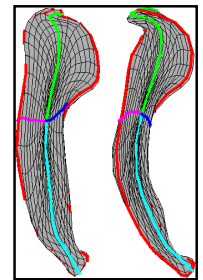

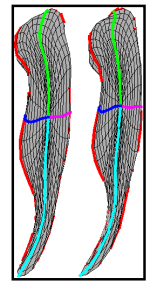

T2A-L / T2B-L T2B-R/T2A-R

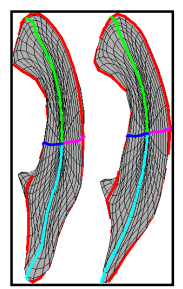

T8A-L / T8B-L T8B-R/T8A-R
Monozygotic twin pairs

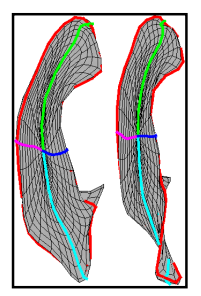

Dizygotic twin pairs

Fig. 3. Illustration of aligned and normalized surfaces of left and right ventricles of $M Z$ (upper row) and DZ (lower row) twin pairs. The parametrization is overlaid as a surface mesh. The color major meridian lines show the stable point-to-point correspondences between surfaces. Visually, shapes of MZ twins are more similar than shapes of DZ twins. Please not that all the shapes are normalized by volume.

10 twin pairs, all healthy controls, to study volume and shape of brain structures. Image data is part of a research of D. Weinberger at NIHM and is published in [Bartley, 1997]. We were interested in the shape variability of the lateral ventricles as ventricular changes are often found to be a marker for disease, e.g. in schizophrenia. We tested the hypothesis that ventricles of $\mathrm{MZ}$ twin pairs are more similar than ventricles of non-identical twin pairs and of unrelated pairs, to corroborate the 
hypothesis that brain structure is significantly controlled by genes. In a first step, we segmented the lateral, third, and forth ventricles by using a statistical classification of voxel intensities and user-guided postprocessing with $3 \mathrm{D}$ connectivity analysis. The variability of volume among the 20 subjects, all healthy controls in the age range of 26-35, was considerable (coefficient of variation $68.8 \%$ ), and there was no significant group difference between $\mathrm{MZ}$ and DZ twin pairs using absolute and relative volume difference as a measure of similarity. However, visual evaluation of 3D renderings of the left and right lateral ventricles (Fig. 3) showed striking similarities in the shape of these structures. To avoid that size differences would dilute or alter the shape measurements, the size of all structures was normalized by volume. We then applied two shape representation methods, surface representation based on spherical harmonics [Kelemen, 1999] and 3D medial mesh representation presented in [Pizer, 1999, Styner, 2000,2001].

\section{Shape analysis using surface representation}

The binary segmentations of the left and right ventricles were parametrized and aligned using the spherical harmonics shape representation method [Kelemen, 1999]. Figure 3 illustrates the resulting surfaces and the parameter meshes. The overlaid of major meridian lines clearly demonstrate the stable point-to-point correspondence obtained by normalizing the parameter space to the poles of the first order ellipsoid. The metric for shape difference is the mean squared distance (MSD) between corresponding surface points. Given the nature of the expansion into harmonics, we can use Parseval's theorem to simply calculate the squared differences between two sets of coefficients. The measures for the MZ and DZ groups were analyzed and compared using standard statistical methods. The square root of the MSD was used for the student's t-tests. The results (Fig. 4) clearly demonstrate that the volume index does not show any group differences, whereas the shape difference of structures normalized for volume is significantly different. $M Z$ shape pairs are more similar $(\mathrm{p}<0.012)$ than DZ shape pairs.
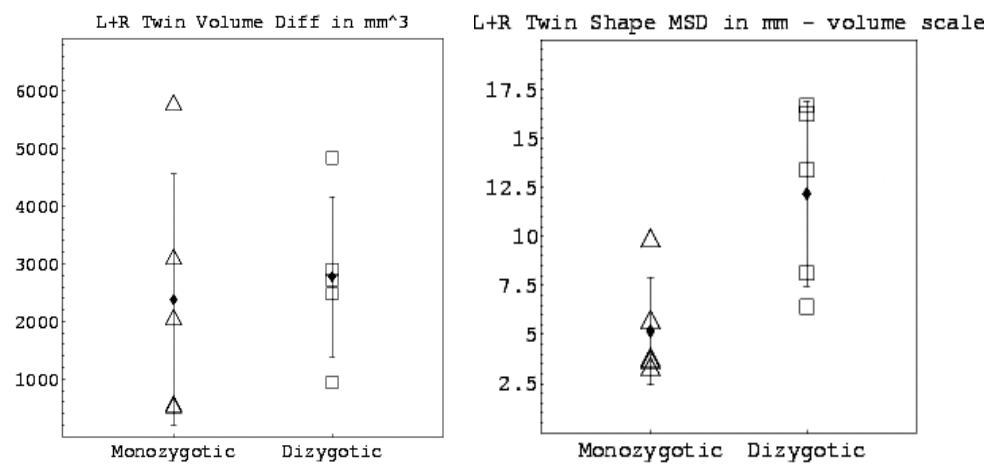

Fig 4. The volume difference index $(\{R-L /(R+L))$ of the ventricles of $M Z$ and $D Z$ twin pairs is nonsignificant $(\mathrm{p}<0.75)$. The shape difference $(\sqrt{ } \mathrm{MSD})$ after volume normalization (right) results in a significantly lower shape difference for $\mathrm{MZ}$ than for $\mathrm{DZ}(\mathrm{p}<0.012)$. 


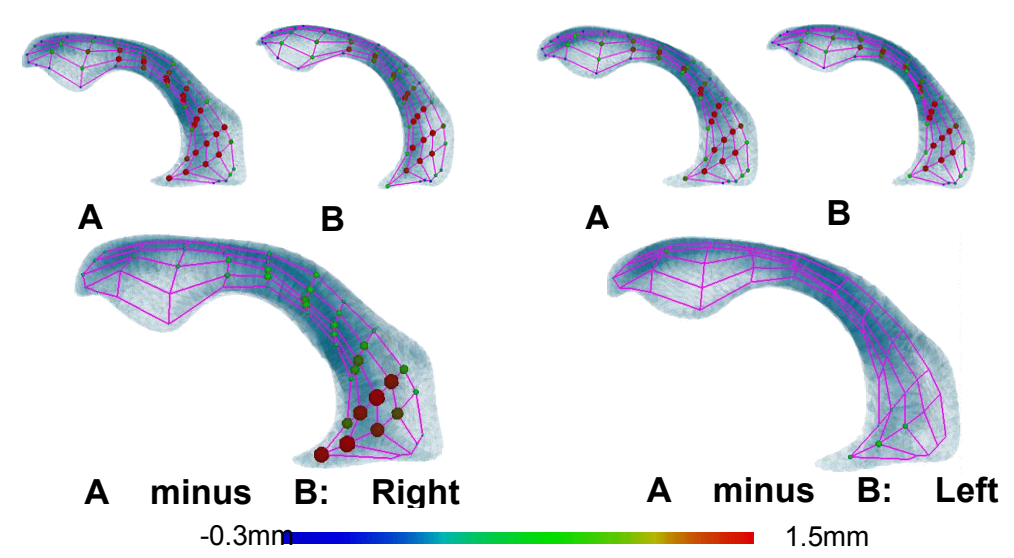

Fig. 5. Shape comparison of ventricles based on medial representations. Right and left lateral ventricles of paired twins A and B are shown in the upper row. The larger figures at the bottom row represent the medial mesh with width (radius) difference at corresponding mesh points. The size and color of the disks indicate local differences between twin A and B in the range of minus $0.3 \mathrm{~mm}$ till $1.5 \mathrm{~mm}$.

\section{Shape analysis using medial mesh representation}

The structures aligned and normalized using the spherical harmonics as described above were processed by deformation of a sampled medial model [Styner and Gerig, 2000,2001]. The mesh of a shape model, derived from a population of ventricular shapes, was deformed to optimally match the new binary structures. The deformation results in a point-to-point correspondence of the discrete set of mesh points (Fig. 5). At each mesh node, we know the position in space and the local width (radius). We used both features independently to test whether the width itself without considering any mesh deformation, or the mesh deformation without considering any width changes, was more significant to find group differences between MZ and DZ twins. Figure 5 and Table 2 demonstrate that the group difference of the width measure alone is highly significant $(\mathrm{p}<0.0065)$ to tell that ventricular shapes in MZ twin pairs are more similar than in DZ pairs. We also tested a third group, which are all the unrelated pairs in our study of 20 subjects (Figure 6). The statistical tests show that the DZ distribution seems to be part of the unrelated pairs distribution. This result is plausible since non-identical twins have the same age and in our study the same gender, which explains the smaller variance of the DZ distribution. The same tests have bee applied to the second feature, the mean absolute distance (MAD) between the mesh node positions. This feature would significantly respond to mesh deformations, e.g. bending, disregarding any local width changes. The results were less significant $(\mathrm{p}<0.035 / 0.011$, Table 2$)$ but present additional shape information. Combined measures using a larger set of local features are currently studies. The interesting shape analysis results have to be compared with the volume analysis presented in the previous section, which was not significant at all, suggesting that shape analysis reveals new information not accessible by simple volume analysis. 


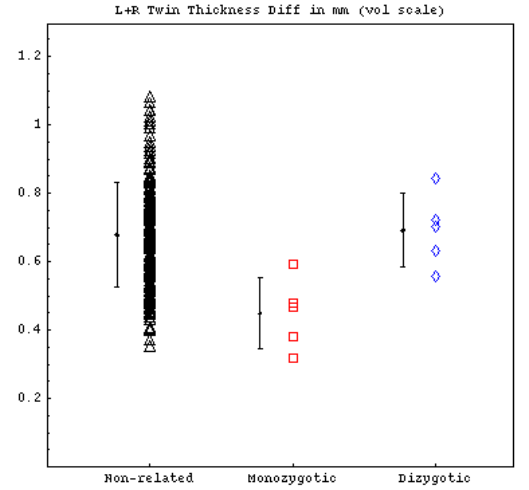

Fig. 6. Statistics of ventricle similarities. The mean absolute width differences at corresponding mesh points is shown for the three groups nonrelated pairs (180 pairs, left), MZ twins (5 pairs, middle) and DZ twins (5 pairs, right). The plots demonstrate that the $\mathrm{DZ}$ twin results could be a subset of the population of unrelated pairs (non significant group difference $\mathrm{p}<0.8562$ ), with smaller variance due to the larger age/gender/sibling similarity. The $M Z$ twins, however, are significantly different from the DZ $(p<0.0065)$ and from the unrelated subjects $(\mathrm{p}<0.0009)$.

\section{Discussion}

This paper clearly demonstrates that shape features provide new information about group differences which is not provided by volume or size only. These results were expected since we intuitively know that structural differences between objects are only poorly defined by volumetric measurements. On the other hand, standard practice in neuroimaging analysis is still volumetry, measuring the brain volume or the volume of subcortical structures and ventricles. We will further extend our new methodology and apply the measurements to data from large clinical studies, in particular to the study of schizophrenia, the enlargment of ventricles in premature infants, to hippocampal measurements in epilepsy, and to autism and Fragile-X studies.

\begin{tabular}{|l|l|l|}
\hline $\begin{array}{l}\text { Width } \\
\text { similarity }\end{array}$ & & \\
\hline & DZ & unrelated \\
\hline MZ & 0.0065 & 0.0009 \\
\hline DZ & & 0.8562 \\
\hline $\begin{array}{l}\text { Location } \\
\text { similarity }\end{array}$ & & \\
\hline & DZ & unrelated \\
\hline MZ & 0.0356 & 0.0110 \\
\hline DZ & & 0.6699 \\
\hline
\end{tabular}

Table 2. Shape similarity of left and right ventricles between MZ/DZ/unrelated pairs using the sampled medial mesh representation. The table shows the p-values for testing mean differences of the width similarity measure (mean absolute radius differences, top) and location similarity measure (mean absolute position difference, bottom).

The comparison of the surface and the medial shape representation with respect to their power of finding group differences clearly demonstrates that the medial representation shows improved significance even using only part of the local 
shape information, here the width at a discrete set of corresponding mesh points. This is equivalent to flattening the mesh and looking only at the width parameter at the sampling points. The mesh deformation itself, independent of the width, can be described as a bending or straightening of figures. In our ventricle study, this measurement was less significant but still below a p-value of $5 \%$. We plan to combine both, width and position, to further improve the discrimination power. A most significant advantage of a sampled medial representation versus a Fourier surface description is the locality of shape information [Yushkevich, 2001], as we can ask for group differences of a part of the mesh only or even at single mesh elements. In epilepsy and schizophrenia, e.g., several groups found significant shape differences using global parametrization or a large set of features. However, such analysis does not give any answer to where and what the pathological differences would be. We will apply our framework to hippocampal and ventricular analysis in studies where group differences were already found, and will test the possibilities for locality and type of shape change given by our new methods.

\section{Acknowledgements:}

We acknowledge D. Weinberger and W. Douglas, NIMH Neuroscience at St. Elizabeth, Washington for providing the twin datasets. We are further thankful to $\mathrm{S}$. Pizer, T. Fletcher and S. Joshi, UNC Computer Science, for providing the m-rep tools for deformable medial meshes.

\section{Bibliography}

Bartley, A.J., Douglas, W.J., and Weinberger, D.R., Genetic variability of human brain size and cortical gyral patterns, Brain (1997), 120, 257-269.

Brechbuehler, Ch, Gerig, G. and Kuebler, O. "Parametrization of closed surfaces for 3D shape description", Comp. Vision and Image Underst. (CVIU), Vol. 61, No. 2, pp. 154-170, 1995

Cootes T.F. et al. (1995) Active Shape Models - Their Training and Application, Computer Vision and Image Understanding, 61(1), pp. 31-59.

Csernansky, J., Joshi, S., Wang, L., Haller, J., Gado, M., Miller, J., Grenander, U., and Miller, M., Hippocampal morphometry in schizophrenia via high dimensional brain mapping, Proc. Natl. Acad. Sci. USA, 95:11406-11411, Sept. 1998

Haller, J., Christensen, G. E., Joshi, S.C, Newcomer, J. W., Miller, M. I., Csernansky, J. G., Vannier, M. W., Hipppocampal MR imaging morphometry by means of general pattern matching, Radiology, Vol. 199, pp. 787-791, 1996.

Haller,J., Banerjee, A., Christensen, G. E, Joshi, S.C.,Miller, M.I., Vannier, M.W., J. Csernansky, G., "Three-Dimensional Hipocampal Volumetry by High Dimensional Transformation of a neuroanatomical Atlas,", Radiology, Vol. 202, pp. 504-510, 1997.

Kelemen, A., Szekely, G. and Gerig, G. (1999) Elastic Model-Based Segmentation of 3D Neuroradiological Data Sets, IEEE Transactions On Medical Imaging, 18, 828-839.

Pizer, S.M., Fritsch, D.S., Yushkevich, P.A., Johnson, V.E., and Chaney, E.L., Segmentation, Registration, and Measurement of Shape Variation via Image Object Shape, IEEE TMI 18(10):851-865, Oct. 1999 
Shenton ME, Wible CG, McCarley RW. (1997) MRI studies in schizophrenia, In: Krishnan KRR, Doraiswamy PM (Eds.), Brain Imaging in clinical Psychiatry. Marcel Dekker, Inc. pp. 297-380.

Staib L.H. and Duncan J.S. (1996) Model-based Deformable Surface Finding for Medical Images, IEEE Trans. Med. Imaging, 15(5), pp. 1-12.

Styner, M. and Gerig, G., Hybrid boundary-medial shape description for biologically variable shapes, Proc. IEEE Workshop on Mathematical Methods in Biomedical Image Analysis (MMBIA), pp. 235-242, June 2000

Styner, M. and Gerig, G., Medial models incorporating object variability for 3D shape analysis, to be published in Proc. of IPMI 2001, Springer Verlag, June 2001

Suddath, R.L., Christison, G.W., Torrey, E.F., Casanova, M.F., and Weinberger, D.R., Anatomical abnormalities in the brains of monozygotic twins discordant for schizophrenia. [Published erratum in New England Journal of Medicine, 322:1616, 1990.], New England Journal of Medicine, 322:789-794, 1990.

Székely, G, A Kelemen, C Brechbühler, G Gerig (1996). Segmentation of 2-D and 3-D objects from MRI volume data using constrained elastic deformations of flexible Fourier contour and surface models. Medical Image Analysis 1(1): 19-34.

Vapnik, Statistical Learning Theory, 1998

Wang, L., Sarang, C.J., Miller, M.I., Grenander, U., and Csernansky, J.G., Statistical Analysis of Hippocampal Asymmetry, in press, NeuroImage. to appear 2001.

Yushkevich, P., Pizer, S.M., Joshi, S., and Marron, J.S., Intuitive, Localized Analysis of Shape Variability, to be published in Proc. IPMI 2001, Springer Verlag, June 2001 